\title{
ASYNCHRONOUS ROTATION IN BY CAM: IT'S GOT A GOOD BEAT AND YOU CAN DANCE TO IT (VERY SLOWLY)
}

\author{
A. D. SILBER
}

University of Washington, Dept. of Astronomy, Box 351580, Seattle, WA 95810-1580, USA

\section{Introduction}

Since its discovery, BY Cam was known to be an atypical AM Her type CV (Remillard et al. 1986). Observations showed the orbital and whitedwarf spin periods differ by about $1 \%$ (Silber et al. 1992). As the white dwarf rotates relative to the secondary, the accretion stream encounters different magnetic geometries, and hence follows different accretion paths to the white dwarf. The magnetic geometry seen by the accretion stream repeats at the beat period $\left(1 / P_{\text {beat }}=1 / P_{\text {spin }}-1 / P_{\text {orb }}\right)$, which is 7 to $20 \mathrm{~d}$ (the range is due to the uncertainty in the measured periods). The shape of the light curve changes from night to night, which can be explained by the changing geometry of the highly-beamed cyclotron emission. Even if the asynchronous rotation is the underlying cause of the changing light curve, the light curve could differ at similar beat phases if the threading process is chaotic. Previous attempts to study the beat period using IUE (Zucker et al. 1995) and optical data (Mason \& Chanmugam 1992) have been inconclusive.

\section{Observations}

Our goal was to observe BY Cam every night for 40 consecutive nights to see if we could detect any sign of the beat period. Observations were carried out at the University of Washington's Manastash Ridge Observatory, Case Western Reserve's Burrell Schmidt, several telescopes at the Crimean Observatory, and the University of Keele's $0.6 \mathrm{~m}$ telescope. We succeeded in obtaining at least one spin period of data on 43 nights over a 65 night span in the fall of 1994 . 


\section{Summary}

We collected a large and well-sampled light curve to study the changes through the beat period. We found a photometric period $(0.137123 \mathrm{~d})$ that is slightly shorter than the spin period determined through polarization studies. This shorter period bolsters the model of BY Cam as an asynchronous rotator (Wynn \& King 1992). We also found a clear, though subtle, sign of the beat period. Once a week the light curve became more complex and the phase shifts relative to the photometric ephemeris. We believe that the accretion stream is switching poles at this phase. If there are two dominant poles this would indicate a beat period of $14 \mathrm{~d}$; three poles suggest a beat period of $21 \mathrm{~d}$, and so on. Though there are clear signs of the beat period at a multiple of $7 \mathrm{~d}$, probably $14 \mathrm{~d}$, there is significant variability of the light curve that does not seem to repeat at this period. Hence the process in which the matter threads the magnetic field and flows to the white dwarf surface may be somewhat chaotic.

Acknowledgments. Data for this work were contributed by Paula Szkody, Don Hoard, Mark Hammergren, Jeff Morgan, Eric Fierce, Knut Olsen, Paul Mason, Robert Rolleston, Eric Ruotsalainen, Elena Pavlenko, Nickolay Shakhovskoy, Sergei Shugarov, Ivan Andronov, Sergey Kolesnikov, Tim Naylor and Ed Schmidt. Additional contributions were made by Paul Mason, Elena Pavlenko, Ivan Andronov and Paula Szkody. Special thanks to George Wallerstein who arranged the MRO telescope schedule to make this project possible.

\section{References}

Mason, P.A., Chanmugam, G., 1992, in "The Vina Del Mar Workshop on Cataclysmic Variable Stars", ed. N. Vogt (San Franscisco: ASP conference series Vol. 29), p203

Remillard, R.A., Bradt, H.V., McClintock, J.E., et al., 1986, Ap. J., 302, L11

Silber, A., Bradt, H.V., Ishida, M., et al., 1992, Ap. J., 389, 704

Wynn, G.A., King, A.R., 1992, MNRAS, 255, 83

Zucker, D.B., Raymond, J.C., Silber, A., 1995, Ap. J., 449, 310 